\title{
Evidence for two energy gaps and Fermi liquid behavior in $\operatorname{SrPt}_{2} \mathrm{As}_{2}$ superconductor
}

\author{
Xiaofeng Xu ${ }^{1 *}$, B. Chen ${ }^{1}$, W. H. Jiao ${ }^{2}$, Bin $\mathrm{Chen}^{3}$, C. Q. Niu ${ }^{1}$, Y. K. Li ${ }^{1}$, J. H. Yang ${ }^{1}$, \\ A. F. Bangura ${ }^{4}$, Q. L. $\mathrm{Ye}^{1}$, C. $\mathrm{Cao}^{1}$, J. H. Dai ${ }^{1}$, Guanghan $\mathrm{Cao}^{2 \dagger}$, and N. E. Hussey ${ }^{5}$ \\ ${ }^{1}$ Department of Physics, Hangzhou Normal University, \\ Hangzhou 310036, China \\ ${ }^{2}$ State Key Lab of Silicon Materials and Department of Physics, \\ Zhejiang University, Hangzhou 310027, China \\ ${ }^{3}$ Department of Physics, \\ University of Shanghai for Science \& Tehcnology , Shanghai, China \\ ${ }^{4}$ RIKEN(The Institute of Physical and Chemical Research), \\ Wako, Saitama 351-0198, Japan. \\ ${ }^{5}$ H. H. Wills Physics Laboratory, \\ University of Bristol, Tyndall Avenue, \\ BS8 1TL, United Kingdom
}

(Dated: July 28, 2021)

\begin{abstract}
We report a detailed calorimetric study on single crystals of the $5 d$-transition metal pnictide SrPt2As2 with a superconducting critical temperature $T_{c} \sim 5 \mathrm{~K}$. The peculiar field dependence of the electronic specific heat coefficient $\gamma$ can be decomposed into two linear components. Moreover, the temperature evolution of the electronic specific heat below $T_{c}$ is best described by a two-gap model. These findings suggest that two energy gaps are associated with the superconductivity. In parallel, we show that the spin-lattice relaxation time $T_{1}$, through nuclear magnetic resonance measurement, obeys the so-called Korringa relation well. This, along with the $T^{2}$ dependence of resistivity at low temperatures, points to a Fermi liquid ground state in this material.
\end{abstract}

A central issue in the field of superconductivity is to elucidate the origin of the pairing interaction, which in turn is intimately related to the pairing symmetry and the gap structure $\Delta(\mathrm{k})$. Notably, nodal $d$-wave superconductivity with $d_{x^{2}-y^{2}}$ pairing symmetry in cuprates is generally believed to originate from the generic spin fluctuations in $\mathrm{CuO}_{2}$ planes 1]. While in iron-based pnictides, the role played by antiferromagnetic spin fluctuations is largely dependent on the strength of the iron $3 d$ electron correlation and remains controversial albeit a sign-reversing $s_{ \pm}$gap structure and multiple energy gaps have been reported[2, 3]. In this regard, it is of fundamental importance to identify the gap structure in understanding the underlying mechanism for the superconducting pairing glue.

Recently, motivated by the discovery of high $T_{c}$ superconductivity in $\mathrm{ThCr}_{2} \mathrm{Si}_{2}$-type pnictides $A \mathrm{Fe}_{2} \mathrm{As}_{2}$ (where $A$ represents alkaline-earth metals) [4], a $5 d$-transition metal platinum-based 122 arsenide $\mathrm{SrPt}_{2} \mathrm{As}_{2}$ was found to be superconducting below $T_{c} \sim 5 \mathrm{~K}[5-7]$. In contrast to other 122 Fe-based superconductors, this ironfree $\mathrm{SrPt}_{2} \mathrm{As}_{2}$ adopts a different $\mathrm{CaBe}_{2} \mathrm{Ge}_{2}$-type structure. Its structure can be viewed as consisting of $\mathrm{Pt}_{2} \mathrm{As}_{2}$ tetrahedral layers alternating with $\mathrm{As}_{2} \mathrm{Pt}_{2}$ layers stacked along the $c$-axis, the former layers with the $\mathrm{Pt}$ ion located in the center of each As tetrahedron and the lat-

\footnotetext{
*Electronic address: xiaofeng.xu@hznu.edu.cn

${ }^{\dagger}$ Electronic address: ghcao@zju.edu.cn
}

ter layer the opposite [8]. Remarkably, in analogy to Febased pnictides, the $\mathrm{SrPt}_{2} \mathrm{As}_{2}$ compound also shows a structural phase transition at $\sim 470 \mathrm{~K}$, which is associated with charge-density-wave (CDW) formation[7]. In spite of these interesting discoveries, the nature of the low-lying quasiparticle excitations and the pairing symmetry have yet to be addressed, in particular the role of the electron-phonon interaction.

In this context, we investigate the superconductivity of single crystals $\mathrm{SrPt}_{2} \mathrm{As}_{2}$ via detailed heat capacity measurement, a bulk probe of the low-lying quasiparticle excitations. Its calorimetric responses, including the field evolution and the temperature dependence of the quasiparticle specific heat, are overall consistent with a scenario of two $s$-wave superconducting gaps opening on different sections of the Fermi surface. We therefore demonstrate that $\mathrm{SrPt}_{2} \mathrm{As}_{2}$, along with the textbook example $\mathrm{MgB}_{2}[9]$, is another prototypical two-gap superconductor. In addition, we show from nuclear magnetic resonance measurement (NMR) that spin fluctuations play only a minor role here. The observation of the Korringa law in this material is consistent with $T^{2}$ resistivity at low temperatures, both pointing to a Fermi liquid ground state.

Single crystals of $\mathrm{SrPt}_{2} \mathrm{As}_{2}$ was synthesized by selfmelting technique, following the procedure described in Ref. 7]. Good single crystallinity of the as-grown samples was then confirmed by x-ray diffraction. For the transport measurements, the sample was cut into a barlike shape with the longest dimension along the basal plane. The specific heat measurement was performed 

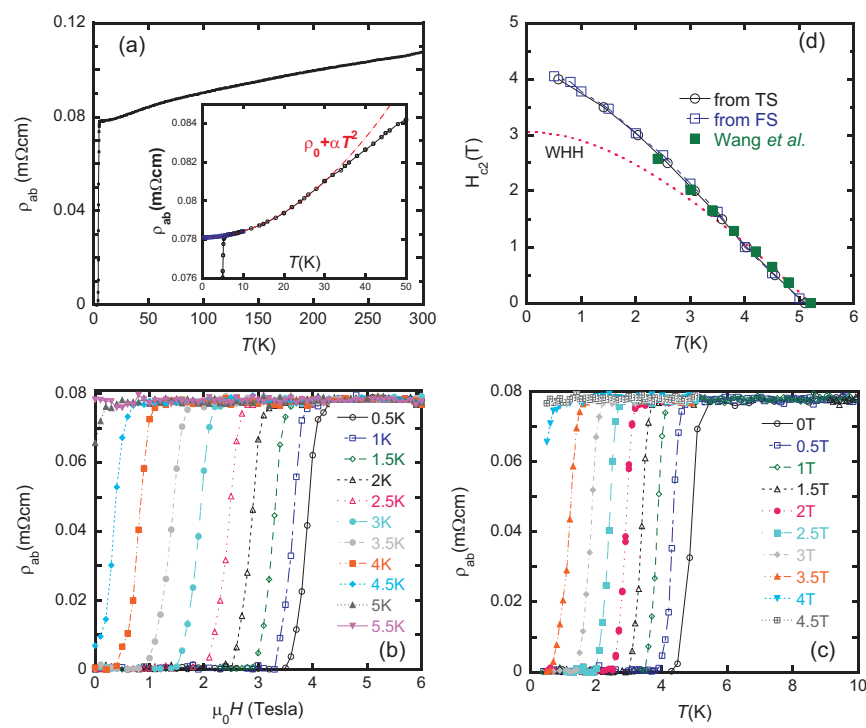

FIG. 1: (Color online) (a) The temperature dependence of the zero-field in-plane resistivity. The inset zooms in the low temperature part with blue squares representing the data extracted from the field sweeps at fixed temperatures (see text). The red line is the fit to $\rho_{0}+\alpha T^{2}$. (b) and (c) show the field sweep (FS) and the temperature sweep (TS) respectively, $H \perp c$, with the extracted $H_{c 2}$ presented in (d), where the data from Fang et al. are also plotted for comparison [7]. The red dashed line in $(\mathrm{d})$ is the WHH fit of $H_{c 2}[10]$.

on a large piece of single crystal of weight $1.3 \mathrm{mg}$ using a commercial Quantum Design PPMS-9 system. The thermometer on the calorimeter puck was well calibrated prior to the measurements in various magnetic fields used in this study between $10 \mathrm{~K}$ down to ${ }^{3} \mathrm{He}$ temperature. The addenda was determined in a separate run. We also performed ${ }^{195} \mathrm{Pt}$ NMR study to investigate the role of spin fluctuations and the Fermi liquid behavior in this material.

Figure 1 encapsulates the zero-field resistivity and the extracted upper critical field $H_{c 2}$ of $\mathrm{SrPt}_{2} \mathrm{As}_{2}$. The temperature dependence of the resistivity is shown in part (a) of this figure and is typical of the behavior reported in the literature, i.e. metallic behavior persisting to low temperature where superconductivity eventually emerges below $T_{c} \sim 5.2 \mathrm{~K}$. The associated $H_{c 2}$ (for $H \perp c$ ) was then determined from both field and temperature sweeps, as shown in Fig.1(b) and Fig.1(c), respectively. The $90 \%$ criterion was used to determine $H_{c 2}$, i.e., the field(temperature) at which $\rho$ reaches $90 \%$ of $\rho_{n}$, the resistivity of the normal state. The as-drawn $H_{c 2}$ was then depicted in Fig.1(d) in which the data from Fang et al. was also reproduced for comparison[7]. Clearly, our data overlap with those of Fang et al. down to the lowest temperature they studied, and persist in going up to 4 Tesla at $0.5 \mathrm{~K}$. We fit our data with conventional one-
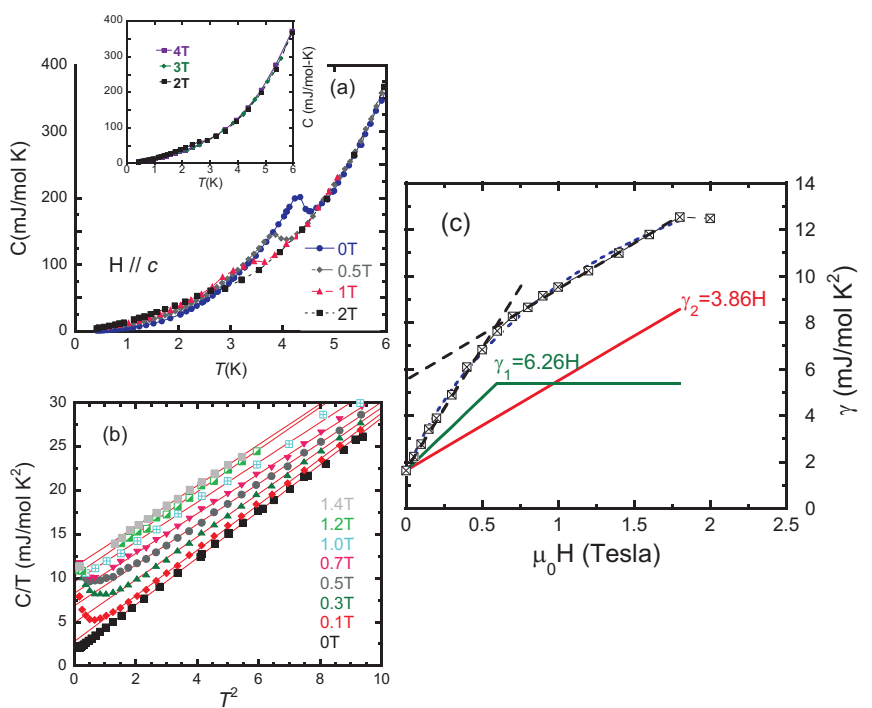

FIG. 2: (Color online) (a) The raw heat capacity data under various fields. The inset shows that the data above 2 Tesla overlap with each other, indicative of the normal state of the sample under these fields. (b) The plot of $C / T$ versus $T^{2}$ enables us to determine the $\gamma$ and $\beta$ as $C / T=\alpha+\beta T^{2}$. The red lines are the fits to this expression. The Debye temperature estimated here is $\sim 160 \mathrm{~K}$, slightly lower than what Fang et al. obtained [7]. (c) shows the $\gamma$ coefficient as a function of field. This dependence was then decomposed into two linear components $\gamma_{1}$ (Green) and $\gamma_{2}$ (Red) with the slope indicated in the Figure. The blue dashed line is the fit to $H \log (c / H)$, corresponding to $\gamma(H)$ for a dirty $d$-wave superconductor.

band Werthamer-Helfand-Hohenberg (WHH) theory [10], as displayed in Fig.1(d), by taking the initial slope near $T_{c},\left.\left(d H_{c 2}^{\perp c} / d T\right)\right|_{T=T c}=0.92 \mathrm{~T} / \mathrm{K}$. However, this one-band WHH theory can not fit our low temperature data satisfactorily. The discrepancy may be ascribed to many reasons, one of which is that the observed superconductivity is not simply from one band but of multi-band nature [11]. Here, we uncover another interesting feature of its normal state transport. As we see from Fig.1(b), there is almost no magnetoresistance in the normal state of $\mathrm{SrPt}_{2} \mathrm{As}_{2}$, evidenced from the flat feature of $\rho(H)$ curves above $H_{c 2}$. Therefore, $\rho(9 T)$ data, or equivalently the intercept at $H=0$ by linearly extrapolating the $\rho_{a b}(T, H)$ above $H_{c 2}[12$, plotted as blue squares in the inset of Fig. 1(a), will represent the genuine normal state resistivity at each temperature indicated. We find that the normal state resistivity of $\mathrm{SrPt}_{2} \mathrm{As}_{2}$ is well fitted by $\rho_{0}+\alpha T^{2}$ up to $T \sim 32 \mathrm{~K}$, a hallmark of the Fermi liquid ground state of a metal.

The specific heat data at different fields are given in Fig.2(a). A clear heat capacity anomaly is observed at $\sim 4.6 \mathrm{~K}$ in zero field. With increasing fields along the $c$ axis, this anomaly is gradually suppressed to lower temperatures and ultimately disappears at $\sim 2$ Tesla. As can be seen in the inset, the data above 2 Tesla all collapse 
into a single curve. The plot of $C / T$ versus $T^{2}$, Fig.2(b), allows us to determine the Sommerfeld coefficient $\gamma$ and phonon contribution $\beta T^{3}$. It is also noted that in the weak fields, there are upturns in $C / T$ at very low temperatures, the origin of which is unknown to us. It may be attributed to the Schottky specific heat due to the Zeeman splitting of the proton nucleus 13]. The resultant $\gamma$ as a function of field is summarized in Fig.2(c).

Fig. 2(c) displays one of the key findings of this paper, namely, the resultant $\gamma$ can be broken down into two linear-in-field lines up to $H_{c 2}$. As can be seen, $\gamma$ is linear in fields up to $\sim 0.6 \mathrm{~T}$ where it changes the slope but keeps growing linearly with fields and finally saturates at $\sim 1.8$ T. It is well known that in a dirty type-II $s$-wave superconductor, $\gamma$ increases linearly with field due to the fact that the thermal excitations mainly arise from the vortex core contribution in $s$-wave superconductor and the number of the vortices grows linearly in $H$. In the clean limit of $d$-wave superconductors, however, $\gamma(H)$ scales as $\sqrt{H}$ owing to the Doppler shift of the quasiparticle spectrum near the line nodes of the gap 14]. Here in $\mathrm{SrPt}_{2} \mathrm{As}_{2}$, neither simple $s$-wave nor clean $d$-wave models explain the observed $\gamma(H)$ curve. Fits to a model of dirty dwave superconductivity, where $\gamma$ scales as $H \log (c / H)$ in weak fields [15], are shown in Fig. 2(c) and work reasonably well. However, this is not compatible with other experimental data as shown below [16]. Instead, reminiscent of other prototypical two-gap superconductors like $\mathrm{MgB}_{2}[9]$ and Chevrel Phases [17], where $\gamma(H)$ shares a wealth of similarities with the present compound, the peculiar shape of $\gamma(H)$ here can also be understood in a scheme of a two-band model. Consequently, in Fig. 2(c), we decomposed $\gamma(H)$ into two linear terms $\gamma_{1}$ and $\gamma_{2}$, with $\gamma_{2}$ linear up to $1.8 \mathrm{~T}$ while $\gamma_{1}$ saturating at $0.6 \mathrm{~T}$. This corresponds to $\frac{\xi_{1}}{\xi_{2}}=\sqrt{\frac{H_{c 2}^{(2)}}{H_{c 2}^{(1)}}}=\sqrt{3}$. Assuming the same Fermi velocities on these two bands, this immediately reveals $\Delta_{2}$ approximately 1.7 times of $\Delta_{1}$. It is also noted that there is a finite $\gamma$ term $\sim 1.6 \mathrm{~mJ} / \mathrm{mol}$ $\mathrm{K}^{2}$ at zero field. We attributed this to arising from the non-superconducting fraction of the sample, which corresponds to $13 \%$ of the total normal carriers. By subtracting this contribution, we end up with the normal state $\gamma$ term for each band equal to $\gamma_{n}^{(1)}=3.7 \mathrm{~mJ} / \mathrm{mol} \mathrm{K}^{2}$ and $\gamma_{n}^{(2)}=6.9 \mathrm{~mJ} / \mathrm{mol} \mathrm{K}^{2}$, respectively.

Considering a fraction of sample still being nonsuperconducting, we subtract the zero-field data with those of $4 \mathrm{~T}$ under which we believe the whole sample enters into the normal state, $\Delta C=C(0 \mathrm{~T})-C(4 \mathrm{~T})$. Since phone heat capacity is field independent, $\Delta C$ removes the phonon contribution and the non-superconducting part, yielding $\Delta C=C_{e s}-\gamma_{s} T$, where $C_{e s}$ is the quasi-particle contribution and $\gamma_{s}$ here means the normal state Sommerfeld coefficient of the superconducting part. As plotted in Fig. 3(a), the heat capacity jump at $T_{c}$ is best
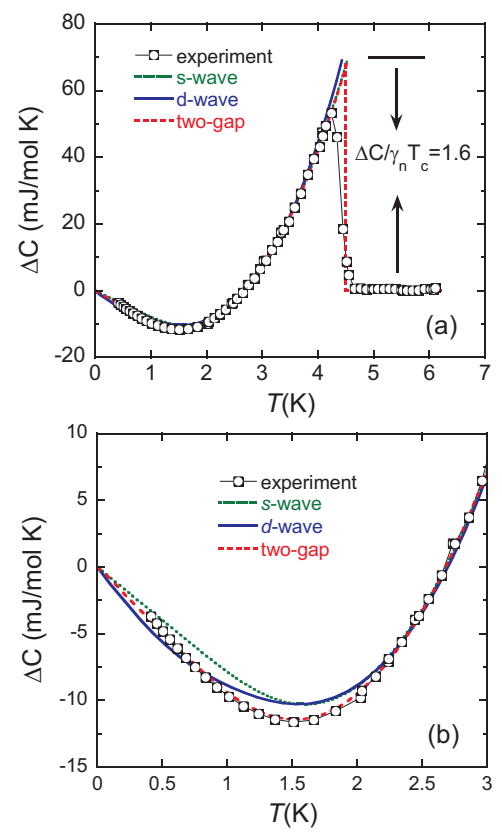

FIG. 3: (Color online) (a) The experimental data of $\Delta C=$ $C(0 \mathrm{~T})-C(4 \mathrm{~T})$ vs $\mathrm{T}$, plotted with the fits using three different gap functions. On this large $T$ scale, three different fits are indistinguishable. However, with the expanded view at low temperatures, panel (b), it is evidently seen the two-gap model fits the experimental data best. The resultant $\Delta C / \gamma_{n} T$ for two-gap model, as indicated in (a), is equal to 1.6, close to the weak-couple BCS value of 1.43 .

resolved in this fashion.

We analyzed our data by fitting $\Delta C$ with different gap functions. In BCS theory, the entropy $S_{e s}$ in the superconducting state is written as 18]

$S_{e s}=-\frac{3 \gamma_{n}}{k_{B} \pi^{3}} \int_{0}^{2 \pi} \int_{0}^{\infty}[(1-f) \ln (1-f)+f \ln f] d \varepsilon d \phi,(1)$

where $\gamma_{n}$ is the normal state $\gamma$, and $f$ stands for the quasi-particle occupation number $f=\left(1+e^{E / k_{B} T}\right)^{-1}$ with $E=\sqrt{\varepsilon^{2}+\Delta^{2}(\phi)} . \quad \Delta(\phi)$ is the angle dependence of the gap function. For conventional $s$-wave, this is angle-independent and for a standard $d$-wave, $\Delta(\phi)=$ $\Delta(T) \cos (2 \phi)$. In addition, we used the well-established $\alpha$ model to describe the temperature dependence of the gap function, in which $\Delta(\phi, T)=\alpha \Delta_{\mathrm{BCS}}(\phi, T)[19]$. Here $\Delta_{\mathrm{BCS}}(\phi, T)$ is the weak coupling BCS gap function. Therefore, the gap is assumed to take the BCS-like form with the magnitude multiplied by a dimensionless parameter $\alpha$, which gives the strength of the (electron-boson) coupling. The specific heat is thereafter calculated by $C_{e s}=T(\partial S / \partial T)$. For the two-gap fitting, two sets of $\gamma_{n}$ and $\alpha$ are used for each gap respectively.

Given the small amount of sample being non- 
TABLE I: The derived fitting parameters using Eqn. 1 for three different gap functions. $\gamma_{n}$ is in the units of $\mathrm{mJ} / \mathrm{mol}$ $\mathrm{K}^{2}$.

\begin{tabular}{|c|c|c|c|c|}
\hline & $T_{C}[\mathrm{~K}]$ & \multicolumn{2}{|c|}{$\alpha$} & $\gamma_{n}$ \\
\hline$s$-wave & 4.6 & \multicolumn{2}{|c|}{1.2098} & 7.97 \\
\hline$d$-wave & 4.8 & \multicolumn{2}{|c|}{1.3088} & 12.23 \\
\hline \multirow{2}{*}{ two-gap } & \multirow{2}{*}{4.6} & $\alpha_{1}$ & $\alpha_{2}$ & \begin{tabular}{|l|l}
$\gamma_{n}^{(1)}$ & $\gamma_{n}^{(2)}$ \\
\end{tabular} \\
\hline & & 0.83508 & 1.2719 & \begin{tabular}{|l|l|}
3.44 & 6.35 \\
\end{tabular} \\
\hline
\end{tabular}

superconducting, we set $\gamma_{n}$ as an adjustable parameter. Hence, the free parameters in the fitting are $\gamma_{n}, \alpha$ and $T_{c}$, similar to the method used in Ref. [18]. At first sight, from Fig.3(a), all three gap functions fit the experimental data equally well. However, the close-up view at the low temperature presented in Fig. 3(b) immediately distinguish these three: The simple $s$-wave could not fit the data satisfactorily nor the $d$-wave gap; Instead, the two $s$-wave gap functions best captures the temperature evolution of $\Delta C$ in the whole temperature range below $T_{c}$. The corresponding fitting parameters are listed in the Table I.

We now examine the two-gap fitting parameters to see its consistency with the picture we got thus far. First of all, the $\gamma$ coefficients for each band obtained from the fitting are 3.44 and $6.32 \mathrm{~mJ} / \mathrm{mol} \mathrm{K}^{2}$ respectively, in agreement with 3.7 and $6.9 \mathrm{~mJ} / \mathrm{mol} \mathrm{K} \mathrm{K}^{2}$ extracted from Fig. 2(c). In addition, the ratio of $\alpha$ (or equivalently $\Delta$ ) values between these two gaps is 1.52 , close to 1.7 obtained above by assuming the same Fermi velocity for each band. The small discrepancy may duly arise from the difference in the Fermi velocities of these two bands. Finally, this two-gap superconductivity is not conflicting with the band structure calculation where the Fermi surfaces consist of electron and hole pockets [8].

Fig. 4(a) shows the Knight shift of ${ }^{195} \mathrm{Pt}$ below 50 $\mathrm{K}$, obtained from the peak resonance field as exemplified at $30 \mathrm{~K}$ in the inset. The $T$-independence of the observed Knight shift indicates the nearly constant spin susceptibility, consistent with the bulk susceptibility measurement which is predominated by $T$-independent Pauli paramagnetism (data not shown here). The spin-lattice relaxation rate $1 / T_{1}$ was measured at the peak position of the field-swept NMR spectrum, and determined by fitting the recovery curve to a single exponential function $\left(\propto \exp \left(-\frac{t}{T_{1}}\right)\right)$ at each fixed temperature. As plotted in Fig. 4(b), $1 / T_{1}$ decreases monotonically with temperature in the whole temperature range studied. The linear temperature dependence of $1 / T_{1}$, known as the Korringa relation $T_{1} T=$ const, is generally believed to arise from the Fermi liquid excitations in a metal. This linear relation yields $T_{1} T=0.23 \mathrm{~s} \mathrm{~K}$ for ${ }^{195} \mathrm{Pt}$. Interestingly, this type of behavior had also been observed in $\mathrm{MgB}_{2}$ by ${ }^{25} \mathrm{Mg}$ and ${ }^{11} \mathrm{~B} \mathrm{NMR}$ measurements [21, 22]. The validity of the Korringa relation in this compound, together with
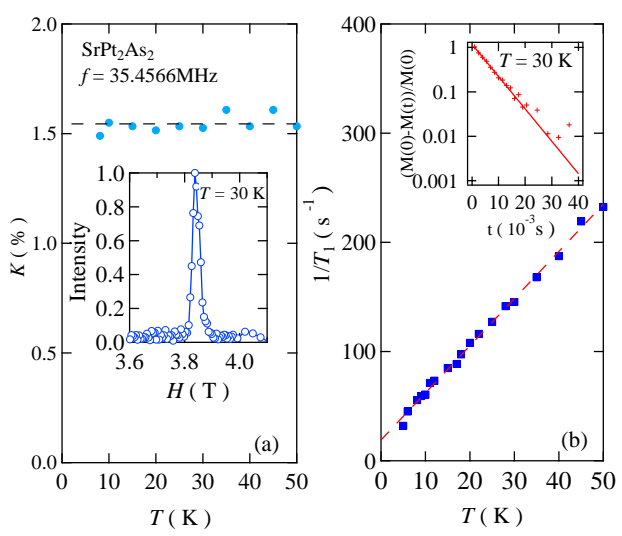

FIG. 4: (Color online) (a) Temperature dependence of ${ }^{195} \mathrm{Pt}$ Knight shift for $\mathrm{SrPt}_{2} \mathrm{As}_{2}$. As an example, the inset shows the field-swept ${ }^{195} \mathrm{Pt}-\mathrm{NMR}$ spectra at $30 \mathrm{~K}$ measured with the fixed frequency of $35.4566 \mathrm{MHz}$. (b) Temperature dependence of the ${ }^{195} \mathrm{Pt}$ nuclear spin-lattice relaxation rate $1 / T_{1}$. The dotted line is the fit to the Korringa relation $T_{1} T=$ const. The inset shows the typical recovery curve measured at $30 \mathrm{~K}$.

its $T^{2}$ resistivity at low temperatures, is indicative of a Fermi liquid ground state.

In summary, based on the experimental probes used in this study, namely, the (magneto)transport, the specific heat as well as the NMR measurements, a coherent picture can be achieved within the framework of twogap, $s$-wave, BCS-like superconductivity developed on the background of a Fermi liquid. The observed superconductivity in this material is seemingly difficult to be associated with any spin fluctuations, while the role of the orbital fluctuations is not clear here. Theoretically, the frustration between the electron-hole interband interaction and the electron-electron intraband scattering can induce a nodal gap [23, 24]. However, this does not seem to be seen here. The observed two-gap energy structure, like the case of $\mathrm{MgB}_{2}$, seems to suggest the coupling between different bands is rather weak [9]. Significantly, it is well known that in $5 d$ electron systems, the Coulomb interaction is relatively weak while the spin-orbit coupling could play an essential role in the electronic structure and their possible superconductivity. Yet, this role may not be always as prominent as previously thought, because it can be weakened near the Fermi surface as evidenced in another platinum-based pnictide $\operatorname{SrPt}_{3} \mathrm{P}[20]$, where the superconductivity with $T_{c} \sim 8.4 \mathrm{~K}$ and strongcoupling to bosonic modes were observed [25]. Finally, the resultant Kadowadi-Woods ratio (KWR) from our measurements is found to be $\sim 20 \mu \Omega \cdot \mathrm{mol}^{2} \cdot \mathrm{K}^{2} / \mathrm{J}^{2}$. This is two orders of magnitude greater than the value typically found for transition metals, and close to those for heavy fermions and strongly correlated oxides. Taken at face value, this potentially indicates strong electronelectron correlations. However, Hussey [27] and Jacko et al. 28] both point out that the absolute value of KWR 
does not necessarily reveal anything about the strength of electronic correlations since material specific parameters (e.g., dimensionality, carrier density, multi-band effects) may be responsible for an enhanced KWR.

The authors would like to acknowledge valuable discussions with Xiaofeng Jin, Y. Matsuda, T. Shibauchi and C. M. J. Andrew, and the technical support from X. X. Yang, H. D. Wang. This work was supported by the National Natural Science Foundation of China.

[1] P. A. Lee, N. Nagaosa, X. G. Wen, Rev. Mod. Phys. 78, 17 (2006).

[2] P. J. Hirschfeld, M. K. Korshunov, and I. I. Mazin, Rep. Prog. Phys. 74, 124508 (2011).

[3] J. Hu and H. Ding, Scientific Rep. 2, 381 (2012).

[4] M. Rotter, M. Tegel, and D. Johrendt Phys. Rev. Lett. 101, 107006 (2008).

[5] A. Imre, A. Hellmann, G. Wenski, J. Grap, D. Johrendt, A. Mewis, Z. Anorg. Allg. Chem. 633, 2037 (2007).

[6] K. Kudo, Y. Nishikubo, M. Nohara, J. Phys. Soc. Jpn. 79, 123710 (2010).

[7] A.F. Fang, T. Dong, H.P. Wang, Z.G. Chen, B. Cheng, Y.G. Shi, P. Zheng, G. Xu, L. Wang, J.Q. Li, and N.L. Wang, Phys. Rev. B 85, 184520 (2012).

[8] I. R. Shein, A. L. Ivanovskii, Phys. Rev. B 83, 104501 (2011).

[9] F. Bouquet, Y. Wang, I. Sheikin, T. Plackowski, and A. Junod, S. Lee and S. Tajima, Phys. Rev. Lett. 89, 257001 (2002).

[10] N. R. Werthamer, E. Helfand, P. C. Hohenberg Phys. Rev. 147, 295 (1966).

[11] F. Hunte, J. Jaroszynski, A. Gurevich, D. C. Larbalestier, R. Jin, A. S. Sefat, M. A. McGuire, B. C. Sales, D. K. Christen, D. Mandrus, Nature 453, 903 (2008).

[12] R A. Cooper, Y. Wang, B. Vignolle, O. J. Lipscombe, S. M. Hayden, Y. Tanabe, T. Adachi, Y. Koike, M. Nohara, H. Takagi, C. Proust, N. E. Hussey, Science 323, 603 (2009).

[13] Y. Nakazawa, H. Taniguchi, A. Kawamoto, and K. Kanoda, Phys. Rev. B 61, R16295 (2000).

[14] Y. Matsuda, K. Izawa and I. Vekhter J. Phys.:Condens. Matter 18, R705 (2006).

[15] N. E. Hussey, Adv. Phys. 51, 1685 (2002).

[16] Also note that in theory, the $H \log (c / H)$ scaling works for dirty $d$-wave superconductors below a crossover field much lower than $H_{c 2}$ (c.f. page 1714 of Ref. [15]). The reasonably good fitting of our experimental data to this model is only coincidence.

[17] A. P. Petrović et al., Phys. Rev. Lett. 106, 017003 (2011).

[18] O. J. Taylor, A. Carrington, J. A. Schlueter, Phys. Rev. Lett. 99, 057001 (2007).

[19] H. Padamsee, J. E. Neighbor, and C. A. Shiffman, J. Low Temp. Phys. 12, 387 (1973).

[20] H. Chen, X. F. Xu, C. Cao, and J. Dai, Phys. Rev. B 86, 125116 (2012).

[21] M. Mali, J. Roos, A. Shengelaya, and H. Keller, and K. Conder, Phys. Rev. B 65, 100518(R) (2002).

[22] C. S. Lue, T. H. Su, B. X. Xie, S. K. Chen, J. L. MacManus-Driscoll, Y. K. Kuo, H. D. Yang, Phys. Rev.
B 73, 214505 (2006).

[23] K. Kuroki, H. Usui, S. Onari, R. Arita, H. Aoki, Phys. Rev. B 79, 224511 (2009).

[24] S. Kasahara, K. Hashimoto, H. Ikeda, T. Terashima, Y. Matsuda, T. Shibauchi, Phys. Rev. B 85, 060503 (2012).

[25] T. Takayama, K. Kuwano, D. Hirai, Y. Katsura, A. Yamamoto, and H. Takagi, Phys. Rev. Lett. 108, 237001 (2012).

[26] K. Kadowaki, S. B. Woods, Solid State Commun. 58, 507 (1986).

[27] N. E. Hussey, J. Phys. Soc. Jpn. 74, 1107 (2005).

[28] A. C. Jacko et al., Nat. Phys. 5, 422 (2009). 